\title{
Un análisis aproximado de la burbuja explosiva generada por liberación instantánea de energía
}

\author{
$1^{\text {st }}$ José Tamagno \\ Dept. Aeronáutica, Universidad Nacional de Córdoba \\ Córdoba, Argentina \\ jose_tamagno@yahoo.com.ar
}

\author{
$3^{\text {rd }}$ Luis Gutiérrez Marcantoni \\ Dept. Aeronáutica, Universidad Nacional de Córdoba \\ Córdoba, Argentina \\ lfgmarcantoni@unc.edu.ar
}

$5^{\text {th }}$ Pascal Bruel

CNRS - University Pau and Pays Adour, LMAP

Pau, France

pascal.bruel@univ-pau.fr

\author{
$2^{\text {nd }}$ Sergio Elaskar \\ Dept. Aeronáutica, Universidad Nacional de Córdoba \\ IDIT, UNC-CONICET \\ Córdoba, Argentina \\ selaskar@unc.edu.ar \\ $4^{\text {th }}$ Juan P. Saldía \\ Dept. Aeronáutica, Universidad Nacional de Córdoba \\ Córdoba, Argentina \\ jsaldia@unc.edu.ar
}

\begin{abstract}
Theoretical concepts and numerical formalisms used to approximately define the explosive bubble that can be generated by instantaneous release of energy are described. This energy is of such magnitude that it supports the use of theoretical concepts of strong shock waves, to determine the limits of bubble expansion. The properties of the gas contained inside, necessary when given the radius of the bubble and its expansion speed, it is desired to quantify the energy for its creation, are determined by applying the similar solution of Sedov and its presentation according to Hayes and Probstein .

Se describen conceptos teóricos y formalismos numéricos utilizados para definir de manera aproximada, la burbuja explosiva que puede generarse por liberación instantánea de energía. Dicha energía es de tal magnitud que avala el uso de conceptos teóricos propios de ondas de choques fuertes, para determinar los limites de expansión de la burbuja. Las propiedades del gas contenido en su interior, necesarias cuando dados el radio de la burbuja y su velocidad de expansión se quiere cuantificar la energía para su creación, son determinadas mediante la aplicación de la solución similar de Sedov y su presentación según Hayes y Probstein.

Index Terms-explosions, shock waves, energy explosiones, ondas de choque, energía
\end{abstract}

\section{INTRODUCCIÓN}

Se pretende que este trabajo se utilize como guía por quienes desean estimar la magnitud y propiedades de la burbuja explosiva que puede ser generada por una liberación instantánea de energía. Se sabe que existen dos posibilidades para definir tal burbuja. Una de ellas mediante la solución numérica de las ecuaciones en derivadas parciales (continuidad, cantidad de movimiento y energía), necesarias para describir el estado y el movimiento del gas contenido en el interior de la burbuja y las condiciones que la limitan [1]. La otra, utilizando soluciones similares y en particular, las propuestas por Howarth y Sedov [2], [3]. Dichas soluciones similares reducen el número de las variables independientes, y para burbujas con simetría esférica las ecuaciones a resolver son transformadas en ecuaciones diferenciales ordinarias. En los cálculos de las propiedades y variables del gas contenido por la burbuja, las expresiones analíticas aquí utilizadas son deducidas de las presentadas en [4], las cuales, son coherentes con la técnica empleada por Sedov. A los efectos de validar el cálculo a efectuar de la energía liberada y su correspondiente burbuja explosiva, se formulan las siguientes hipótesis:

1) Las propiedades del flujo ambiental en reposo (aire), son conocidas y permanecen constantes.

2) La velocidad de expansión de la burbuja es tan elevada que la relación entre las densidades del flujo detrás y adelante de la discontinuidad ( $u$ onda de choque) que limita a la burbuja respecto del aire ambiental, se aproxima al valor máximo permisible (si la relación de calores específicos tanto para el gas externo a la burbuja como el de su interior es 1.4, dicho valor máximo es 6). Esta aproximación al valor máximo de la relación entre las densidades de ambos lados, convalida la utilización de las soluciones similares ya citadas y por consiguiente a las expresiones analíticas formuladas en [4], necesarias para cuantificar los parámetros que definen las propiedades y variables del gas dentro de la burbuja.

La utilización de las relaciones entre las magnitudes físicas de la corriente fluida en ambos lados del límite de la burbuja explosiva, amerita la descripción de conceptos básicos que hacen a la discontinuidad existente entre el dominio interno de la burbuja y el externo o ambiental. Tales conceptos conllevan a postular la formación de ondas de choque fuertes.

\section{Conceptos bÁsicos Sobre OndAS DE CHOQUe FUERTES}

Se sabe que en todo flujo de un gas ideal y perfecto (y sin tener en cuenta la radiación), la entropía permanece 
constante sobre cada línea de corriente aunque puede variar desde una línea a otra. Esta consideración es válida mientras no se presenten fenómenos que introduzcan cambios abruptos (discontinuidades), en las propiedades del flujo [5], [6]. Estos tipos de fenómenos suelen producirse en flujos supersónicos. En efecto, se ha comprobado experimentalmente que cuando la velocidad del flujo gaseoso es supersónica se pueden producir cambios muy rápidos en sus propiedades. Esto cambios ocurren en distancias tan pequeñas comparadas con una dimensión característica del movimiento, que desde el punto de vista de las aplicaciones prácticas pueden ser considerados como discontinuidades. Estas discontinuidades a través de las cuales se produce una variación finita de las magnitudes físicas del movimiento se denominan ondas de choque. La presencia de ondas de choque en un fluido ideal y perfecto, son causantes de evoluciones no isoentrópicas a lo largo de las líneas de corriente del movimiento.

El análisis que sobre las ondas de choque se presenta a continuación tiene por objeto describir las relaciones existentes entre las magnitudes físicas de la corriente fluida en ambos lados de la discontinuidad y son aplicables únicamente a ondas de choque fuertes de gran intensidad y normales a la dirección del flujo.

Se supone que la burbuja explosiva existe y la discontinuidad $\mathrm{u}$ onda de choque que la limita, es decir separa el flujo interno del externo, es esférica y tiene un radio $r_{b}$ En la intersección de la superficie esférica de la burbuja con su radio se puede considerar que la onda de choque es normal respecto del radio y por lo tanto son aplicables las relaciones entre las variables físicas que caracterizan al estado adelante y detrás de una onda de choque plana. Considérese entonces, la propagación de una onda de choque plana en un gas en reposo cuya presión es $p_{0}$, su densidad $\rho_{0}$ y su energía interna por unidad de masa es $E_{i(0)}$. Se indican con $U$ a la velocidad de la onda de choque e inmediatamente detrás con $u_{s}, \rho_{s}, p_{s}$ y $E_{i(s)}$ a la velocidad, densidad, presión y a la energía interna por unidad de masa, respectivamente.

La continuidad entre ambos lados de la onda de choque se puede expresar por

$$
\rho_{0} U=\rho_{s}\left(U-u_{s}\right)
$$

de donde se deduce, luego de introducir $g_{s}=\rho_{0} / \rho_{s}$, que

$$
U-u_{s}=U g_{s}
$$

La relación entre las cantidades de movimiento adelante y detrás de la onda de choque, se puede escribir como se muestra a continuación:

$$
p_{0}+m U=p_{s}+m\left(U-u_{s}\right)
$$

Si $p_{s} \gg p_{0}$ la Ec. (3) se reduce a:

$$
p_{s}=m u_{s}=\rho_{0} U u_{s}
$$

Si se utiliza la energía interna $\left(E_{i}=c_{v} T\right)$ por unidad de masa, la ecuación de la energía aplicable a ambos lados de la onda de choque se puede escribir [5]. Note que la dimensión de la ecuación de la energía, Ec. (5), es $\mathrm{J} / \mathrm{kg}$.

$$
E_{i(s)}+\frac{p_{s}}{\rho_{s}}+\frac{1}{2}\left(U-u_{s}\right)^{2}=E_{i(0)}+\frac{p_{0}}{\rho_{0}}+\frac{1}{2} U^{2}
$$

Luego de tener en cuenta las Ecs. (2) y (4), se obtiene:

$$
2\left(E_{i(s)}-E_{i(0)}\right) \rho_{0}=2 p_{0}+p_{s}\left(1-g_{s}\right)
$$

y si la intensidad del choque es tal que $p_{s} \gg p_{0}, E_{i(s)} \gg$ $E_{i(0)}$, la Ec. (5) se reduce a

$$
2 \rho_{0} E_{i(s)}=p_{s}\left(1-g_{s}\right)
$$

Si el gas es perfecto y la relación de calores específicos $\gamma$ se mantiene constante, la energía interna por unidad de masa puede conectarse con la presión y la densidad mediante la expresión

$$
E_{i}=\frac{1}{(\gamma-1)} \frac{p}{\rho}
$$

luego de tener en cuenta que $R=c_{p}-c_{v}$. Por lo tanto, para el gas inmediatamente detrás del choque se puede hacer:

$$
E_{i(s)}=\frac{1}{(\gamma-1)} \frac{p_{s}}{\rho_{s}}
$$

Introduciendo el valor de $E_{i(s)}$ dado por Ec. (9) en la ecuación de la energía dada por Ec (7), se consigue

$$
g_{s}=\frac{\rho_{0}}{\rho_{s}}=\frac{\gamma-1}{\gamma+1}
$$

Si ahora se tiene en cuenta a Ec. (1), se puede expresar el valor de $u_{s}$ por

$$
u_{s}=\frac{2}{\gamma+1} U
$$

y combinando Ecs. (4) y (11) se puede relacionar $p_{s}$ con $U$, resultando:

$$
p_{s}=\frac{2}{\gamma+1} \rho_{0} U^{2}
$$

Desde la Ec. (7), expresando $p_{s}$ y $g_{s}$ en términos de $U$ y $u_{s}$, se tiene que:

$$
E_{i(s)}=\frac{1}{2} u_{s}^{2}
$$

y mediante Ec. (11) es posible relacionar $E_{i(s)}$ con $U$ :

$$
E_{i(s)}=\frac{2}{(\gamma+1)^{2}} U^{2}
$$

Es de notar desde la Ec. (10), que la relación entre densidades a través de la onda de choque es independiente de las presiones cuando $p_{s} \gg p_{0}$. 


\section{El ANÁlisis DE LA BURBUJA}

En primer lugar se describirá como mediante una constante de proporcionalidad denominada $\beta$, pueden relacionarse el radio $r_{b}$ de la burbuja con la energía total $E$ liberada, con el tiempo $t_{b}$ de expansión de la burbuja desde un punto explosivo inicial hasta el radio $r_{b}$, y con la densidad del fluido externo.

Téngase en cuenta que la dimensión de la energía total es el Joule $(J)$, mientras que la de $E_{i}$ por unidad de masa es el $J / k g$.

Luego se evaluará como se distribuyen las propiedades características (presión y densidad), y la velocidad del gas contenido en su interior utilizando la metodología de soluciones similares propuesta por Sedov [3]. Finalmente, se procederá con el cálculo de la energía total de la burbuja en términos de las energías cinética y térmica del gas contenido, y con la determinación del valor de la constante $\beta$.

\section{A. La energía a liberar necesaria para crear la burbuja}

Las propiedades del fluido en reposo (aire), que está delante de la burbuja son típicas y se mantienen constantes. Por lo tanto, se consideran datos los siguientes valores: $p_{0}=101325 \mathrm{~Pa} ; \rho_{0}=1.221 \mathrm{~kg} / \mathrm{m}^{3} ; T_{0}=288 \mathrm{~K}$;

$a_{0}=340 \mathrm{~m} / \mathrm{s}$ y $\gamma=1.4$.

Si se tiene en cuenta que la energía total $E$ dentro de la burbuja se conserva, su radio se puede estimar mediante la siguiente expresión que desde un punto de vista dimensional es correcta:

$$
r_{b}=\beta\left(\frac{E}{\rho_{0}} t_{b}^{2}\right)^{1 / 5}
$$

siendo $\beta$ una constante sin dimensiones a determinar, $t_{b}$ el tiempo que transcurre desde el instante inicial de la liberación de la energía y la expansión de la burbuja hasta alcanzar el radio $r_{b}$. De la Ec. (15) se puede explicitar la energía total $E$, obteniéndose:

$$
E=E_{c i n}+E_{\text {term }}=\beta^{-5} \rho_{0} \frac{r_{b}^{5}}{t_{b}^{2}} \quad(J)
$$

La velocidad del choque o de expansión de la burbuja $(U)$ también se puede obtener derivando respecto del tiempo la Ec. (15):

$$
U=\frac{d r_{b}}{d t}=\frac{2}{5} \beta\left(\frac{E}{\rho_{0}}\right)^{1 / 5} t_{b}^{-3 / 5}=\frac{2}{5} \frac{r_{b}}{t_{b}}
$$

Si se asume un valor de $r_{b}$ y se acepta un valor $U$ asignado a la velocidad de expansión de la burbuja, el tiempo $t_{b}$ puede computarse a partir de:

$$
t_{b}=\frac{2}{5} \frac{r_{b}}{U}
$$

Si el tiempo $t_{b}$ que aparece en la Ec. (16) que define la energía liberada, se reemplaza según indica la Ec. (18), se consigue:

$$
E=\frac{25}{4} \beta^{-5} \rho_{0} U^{2} r_{b}^{3}
$$

El cálculo de la energía total $E$, implica la selección de una velocidad $U$ congruente con la hipótesis de choque fuerte y la evaluación de la constante $\beta$.
Con el propósito de convalidar las hipótesis de la onda de choque fuerte que limita a la burbuja y ser aplicables las aproximaciones definidas por las Ecs. (10) (11), (12), (13) y (14), se acepta que el número de Mach asociado con la velocidad de expansión de la burbuja debe ser igual o mayor que 10 (Mach $\geq 10$ ). Entonce, la velocidad de expansión de la burbuja debe ser superior a $3400 \mathrm{~m} / \mathrm{s}$ si la velocidad del sonido del aire en reposo es $a_{0}=340 \mathrm{~m} / \mathrm{s}$. Por lo tanto si se establece $U=3500 \mathrm{~m} / \mathrm{s}$, resulta aplicable la Ec. (12) y el valor de la presión después del choque es $p_{s}=12.4644 \mathrm{MPa}$. La relación de presiones a través del choque que limita a la burbuja resulta ser $\frac{p_{s}}{p_{0}}=123.0141$

Para el cálculo de la relación entre las temperaturas adelante e inmediatamente detrás del choque, puede utilizarse la siguiente aproximación:

$$
\frac{T_{s}}{T_{0}}=\frac{p_{s}}{p_{0}} \frac{\gamma-1}{\gamma+1}=123.0141\left(\frac{1}{6}\right)_{\gamma=1.4}=20.5023
$$

y la temperatura $T_{s}=288 \times 20.5023=5905 \mathrm{~K} . \mathrm{Se}$ destaca que con $\mathrm{Mach}=10$, la relación entre densidades $\rho_{s} / \rho_{0}$ se aproxima en un $95 \%$ al valor máximo permisible (6 si $\gamma=1.4)$.

Es de interés evaluar la energía interna $E_{i(s)}$ que resulta de aplicar la Ec. (14). Se obtiene $E_{i(s)}=4.2535 \mathrm{MJ} / \mathrm{kg}$.

\section{B. Aproximación de Sedov}

Para determinar el valor de la constante $\beta$, es necesario formular el cálculo de la energía total $E$ liberada en términos de las energías cinética y térmica del gas contenido por la burbuja. Dicha formulación requiere evaluaciones locales de la densidad, la presión y de la velocidad en su interior. Para su realización, se presentan dos alternativas: una de ellas es utilizar la metodología de soluciones similares [?], [3] y la otra, solucionar numéricamente las ecuaciones diferenciales de Euler que son aplicables cuando la simetría de la burbuja explosiva es esférica [1]. Las citadas ecuaciones de Euler son:

$$
\begin{gathered}
\frac{\partial \rho}{\partial t}+\frac{\partial}{\partial r}(\rho v)+\frac{2}{r} \rho v=0 \\
\frac{\partial v}{\partial t}+v \frac{\partial v}{\partial r}+\frac{1}{\rho} \frac{\partial p}{\partial r}=0 \\
\frac{\partial}{\partial t}\left(p \rho^{-\gamma}\right)+v \frac{\partial}{\partial r}\left(p \rho^{-\gamma}\right)=0
\end{gathered}
$$

donde $r$ es la única coordenada espacial y $v$ la velocidad en la dirección de $r$. La Ecs. (21), (22) y (23) son la de continuidad, cantidad de movimiento y energía respectivamente. Es de notar que la estricta aplicación del concepto de liberación instantánea de la energía (punto explosivo), puede introducir dificultades en la formulación de las condiciones iniciales adecuadas para la solución numérica de las Ecs. (21), (22) y (23).

El procedimiento a seguir para la obtención de las soluciones similares a partir de las ecuaciones de Euler, consiste en reemplazar las variables dependientes en las Ecs. (21), (22) y (23) por nuevas variables sin dimensiones y luego, lograr 
que estas últimas puedan expresarse en términos de solo una nueva y única variable independiente también sin dimensión. Estos procesos conducen a que el sistema de ecuaciones con diferenciación parcial original se reemplace por un sistema de ecuaciones diferenciales ordinarias independientes del tiempo, las cuales pueden ser resueltas en forma exacta siguiendo a Sedov [3]. Las soluciones similares para las variables $r / r_{s}$, $u / u_{s}, \rho / \rho_{s}$ y $p / p_{s}$ que se muestran a continuación, son deducidas de las presentadas por Hayes y Probstein [4]:

$$
\begin{gathered}
\ln \frac{r}{r_{b}}=-k \ln \left(F_{1}\right)+\frac{k(\gamma-1)}{2 \gamma-\phi} \ln \left(F_{2}\right)-\Phi \ln \left(F_{4}\right) \\
\ln \frac{u}{u_{s}}=(1-k) \ln \left(F_{1}\right)+\frac{k(\gamma-1)}{2 \gamma-\phi} \ln \left(F_{3}\right)-\Phi \ln \left(F_{4}\right) \\
\ln \frac{\rho}{\rho_{s}}=-\frac{6 k}{2-\phi} \ln \left(F_{2}\right)+\frac{3 k}{2 \gamma-\phi} \ln \left(F_{3}\right)+ \\
\begin{aligned}
\ln \frac{p}{p_{s}}=2(1-k) \ln \left(F_{1}\right) & +\left(1-\frac{6 k}{2-\phi}\right) \ln \left(F_{4}\right) \\
& +\left(-2+\frac{6}{2-\phi}\right) \Phi \ln \left(F_{2}\right)
\end{aligned}
\end{gathered}
$$

El valor del factor $k$ que aparece con frecuencia en los coeficientes de los logaritmos naturales de las funciones $F_{i}$ es $2 / 5$, debido a la simetría de la burbuja supuesta esférica.

Las funciones $F_{i}(i=1,2,3,4)$, en términos de la única variable independiente a la cual se llega con las soluciones similares y que se denominará $V$, se definen a continuación:

$$
\begin{gathered}
F_{1}(V)=\frac{\gamma+1}{2} V \\
F_{2}(V)=\frac{\gamma+1}{\gamma-1}(1-V) \\
F_{3}(V)=\frac{\gamma+1}{\gamma-1}(\gamma V-1) \\
F_{4}(V)=\frac{\gamma+1}{\gamma+1-\phi}\left(1-\frac{\phi}{2} V\right)
\end{gathered}
$$

Los parámetros $\phi$ y $\Phi$, utilizados tanto en las Ecs. (24), (25), (26), (27), como en la Ec. (31), están dados por:

$$
\begin{gathered}
\phi=\frac{2}{5}[\gamma+1+2(\gamma-1)] \\
\Phi=\frac{2}{5}\left(\frac{\gamma+1-\phi}{\phi}+\frac{\gamma-1}{2 \gamma-\phi}\right)
\end{gathered}
$$

Es de observar que cuando $V=2 /(\gamma+1)$ las funciones $F_{i}$ $(i=1,2,3,4)$ utilizadas en las ecuaciones para las soluciones de las variables $r / r_{b}, u / u_{s}, \rho / \rho_{s}$ y $p / p_{s}$ toman el valor 1 inmediatamente detrás del choque. Si $V=1 / \gamma$, la función $F_{3}=0$, lo cual hace que $r / r_{b}=0$. No obstante, por no depender de $F_{3}$ la relación $p / p_{s} \neq 0$ cuando $r / r_{s}=0$. Por tanto, en el procedimiento para encontrar las valores de las variables $r / r_{b}, u / u_{s}, \rho / \rho_{s} \mathrm{y} p / p_{s}$ en el interior de la burbuja explosiva, los valores del intervalo de variación de la variable de similitud $V$ son dados por:

$$
\frac{1}{\gamma} \leq V \leq \frac{2}{\gamma+1}
$$

\section{Evaluación de la constante $\beta$}

La energía total $E$ que debe ser liberada por la explosión expresada en términos de las propiedades y variables del flujo en el interior de la burbuja, es computable a partir de las integrales

$$
E=4 \pi\left[\int_{0}^{r_{s}} \frac{p}{\gamma-1} r^{2} d r+\frac{1}{2} \int_{0}^{r_{s}} \rho u^{2} r^{2} d r\right]
$$

donde la primera integral es la energía térmica $\left(E_{\text {term }}\right)$ y la segunda la cinética $\left(E_{c i n}\right)$.

La integral de la energía térmica se puede escribir como se indica a continuación:

$$
\begin{aligned}
\frac{E_{\text {term }}}{4 \pi r_{s}^{3}} & =\frac{p_{s}}{(\gamma-1)} \int_{0}^{1} \frac{p}{p_{s}} \xi^{2} d \xi \\
& =\frac{2}{(\gamma+1)(\gamma-1)} \rho_{0} U^{2} \int_{0}^{1} \frac{p}{p_{s}} \xi^{2} d \xi
\end{aligned}
$$

donde $p_{s}$ es la presión inmediatamente detrás del choque que limita a la burbuja, $r_{s}=r_{b}$ el radio de la burbuja y por simplicidad se hace $\xi=r / r_{s}$. También se ha reemplazado $p_{s}$ fuera de la integral por $2 /(\gamma+1) \rho_{0} U^{2}$.

Procediendo de manera semejante a lo realizado con la integral de la energía térmica, la integral de la energía cinética se puede escribir como se indica a continuación:

$$
\begin{aligned}
\frac{E_{\text {cin }}}{4 \pi r_{b}^{3}} & =\frac{1}{2} \rho_{s} u_{s}^{2} \int_{0}^{1} \frac{\rho}{\rho_{s}} \frac{u^{2}}{u_{s}^{2}} \xi^{2} d \xi \\
& =\frac{2}{(\gamma+1)(\gamma-1)} \rho_{0} U^{2} \int_{0}^{1} \frac{\rho}{\rho_{s}} \frac{u^{2}}{u_{s}^{2}} \xi^{2} d \xi
\end{aligned}
$$

donde se ha hecho uso de las relaciones $\rho_{s} u_{s}^{2}=\frac{4 \rho_{0} U^{2}}{(\gamma+1)(\gamma-1)}$, y $\rho_{s} / \rho_{0} \sim(\gamma+1) /(\gamma-1)$.

Entonces, la energía $E$ que debe ser liberada se puede expresar de la manera siguiente:

$$
\frac{E}{r_{b}^{3} \rho_{0} U^{2}}=\frac{4 \pi}{\kappa}\left[\int_{0}^{1} \frac{p}{p_{s}} \xi^{2} d \xi+\int_{0}^{1} \frac{\rho}{\rho_{s}} \frac{u^{2}}{u_{s}^{2}} \xi^{2} d \xi\right]
$$

donde $\kappa=(\gamma+1)(\gamma-1)$ y el segundo miembro de esta es adimensional. A partir de la Ec.(19) se consigue:

$$
\beta^{-5}=\left(\frac{2}{5}\right)^{2} \frac{1}{r^{3} \rho_{0} U^{2}} E
$$

Si se tiene en consideración la Ec. (38), la constante $\beta$ puede ser evaluada como:

$$
\beta=\left[\left(\frac{4}{5}\right)^{2} \pi \frac{1}{(\gamma+1)(\gamma-1)}(I+I I)\right]^{-1 / 5}
$$

donde $I$ y $I I$ representan respectivamente, primera y segunda integrales que contiene la Ec. (38). Finalmente, se obtiene: $\beta=1.1918$ 


\section{La energía liberada en función del radio de la burbuja}

$\mathrm{Si}$ se asume el radio de la burbuja $r_{b}$ y se determina el tiempo $t_{b}$ mediante la Ec. (18) puesto que la velocidad $U=3500 \mathrm{~m} / \mathrm{s}$ (así definida para garantizar la existencia de un choque fuerte), se puede utilizar la Ec. (19) para determinar el valor de la energía total $E$ a liberar necesaria para construir diversas burbujas explosivas con velocidad del choque $U=3500 \mathrm{~m} / \mathrm{s}$. Valores de dichas energías están listados en la tercera columna de la Tabla 1.

\begin{tabular}{|c|c|c|c|}
\hline$r_{b}(m)$ & $t_{b}(s)$ & $E(M J)$ & $T N T(k g)$ \\
\hline \hline 0.75 & $85.71 \cdot 10^{-6}$ & 16.402 & 3.920 \\
\hline 1.00 & $114.29 \cdot 10^{-6}$ & 38.879 & 9.292 \\
\hline 2.00 & $228.57 \cdot 10^{-6}$ & 311.032 & 74.338 \\
\hline 4.00 & $457.14 \cdot 10^{-6}$ & 2488.256 & 594.707 \\
\hline 6.00 & $691.4 \cdot 10^{-6}$ & 8397.864 & 2007.138 \\
\hline 10.00 & $1152.2 \cdot 10^{-6}$ & 38879.000 & 9292.304 \\
\hline
\end{tabular}

Tabla 1. Energía a liberar en forma instantánea necesaria para la creación de una burbuja explosiva, dados el radio y el tiempo de formación.

Los datos presentados en la Tabla 1, son aplicables si la velocidad de expansión de la onda es $3500 \mathrm{~m} / \mathrm{s}$ (Mach= 10.294). Por lo tanto, las condiciones detrás de la onda de choque son las mismas para todos los casos puesto que las condiciones del medio en el cual se propaga la onda expansiva también lo son. Las relaciones entre la energía total liberada $E$ y la cantidad en kilogramos del explosivo TNT (Trinitrotolueno) necesaria, se obtienen a partir del dato que $1 \mathrm{~kg}$ de TNT libera $4.184 \mathrm{MJ}$. La presión $p_{s}$ y la temperatura $T_{s}$, inmediatamente detrás del choque que limita a la burbuja han sido previamente calculadas y sus respectivos valores son 12.4644 MPa y $5905 \mathrm{~K}$.

Cabe señalar, que las energías liberadas también pueden calcularse utilizando en la Ec. (19) el valor de $E_{i(s)} \rho_{s}$ en lugar de $\rho_{0} U^{2}$. Como ambas expresiones tienen la misma dimensión $\left(\mathrm{J} / \mathrm{m}^{3}\right)$, pueden relacionarse a través de una constante identificada aquí como $C$. Por lo tanto para $\gamma=1.4$ se tiene:

$$
C=\frac{\rho_{0} U^{2}}{E_{i(s)} \rho_{s}}=\frac{\left(\gamma^{2}-1\right)}{2}=0.48
$$

Entonces, Ec. (19) se puede escribir:

$$
E=\frac{25}{4} \beta^{-5} C E_{i(s)} \frac{\rho_{s}}{\rho_{0}} \rho_{0} r_{s}^{3}
$$

E. La distribución de la temperatura absoluta dentro de la burbuja

La relación entre las temperaturas $T$ existentes en el interior de la burbuja y la determinada inmediatamente detrás de la onda de choque $T_{s}$, es decir la relación $T / T_{s}$ se puede calcular utilizando las correspondientes ecuaciones de estado:

$$
\frac{T}{T_{s}}=\frac{p}{p_{s}} \frac{\rho_{s}}{\rho}
$$

Esta ecuación escrita con términos logarítmicos dados por las Ecs. (26) y (27) resulta ser:

$$
\ln \frac{T}{T_{s}}=2(1-k) \ln F_{1}+\ln F_{2}-\frac{3 k}{2 \gamma-\phi} \ln F_{3}-2 \Phi \ln F_{4}
$$

Si se escribe $T / T_{s}$ sin utilizar logaritmos naturales, se obtiene la solución similar dada en [4]:

$$
\frac{T}{T_{s}}=F_{1}^{2(1-k)} F_{2} F_{3}^{-\frac{3 k}{2 \gamma-\phi}} F_{4}^{-2 \Phi}
$$

Se constata que por ser la función $F_{3}(V)$ nula si $V=1 / \gamma$ (ver Ec. (30)), y el exponente permanece negativo $(2 \gamma-\phi=$ 1.52 con $\gamma=1.4$ ), la relación $T / T_{s} \rightarrow \infty$. Este valor de la temperatura $T$ cuando $r \rightarrow 0$, es consistente con la hipótesis fundamental conducente al análisis de Sedov: liberación total e instantánea de la energía desde un punto inicial explosivo situado en el centro de la burbuja y luego, considerada constante durante la formación de dicha burbuja.

Como los valores de $p / p_{s}$ y $\rho / \rho_{s}$ dentro de la burbuja se pueden expresar como funciones de $r / r_{s}$, también pueden ser lo los valores de la relación entre las temperaturas $T / T_{s}$. Se puede entonces, construir la tabla de valores que se muestra a continuación:

\begin{tabular}{|c|c|c|}
\hline$V$ & $\frac{r}{r_{s}}$ & $\frac{T}{T_{s}}$ \\
\hline \hline $2 /(\gamma+1)$ & 1,0 & 1,0 \\
\hline 0.82 & 0.9858 & 1.1444 \\
\hline 0.80 & 0.9621 & 1.4220 \\
\hline 0.76 & 0.8981 & 2.5255 \\
\hline 0.72 & 0.7211 & 13.5869 \\
\hline 0.715 & 0.5793 & 70.4702 \\
\hline 0.7143 & 0.3839 & 1546.395 \\
\hline $1 / \gamma$ & 0.0 & $\infty$ \\
\hline
\end{tabular}

Tabla 2: Distribución de la temperatura $T$ en el interior de la burbuja explosiva.

La Tabla 2, predice que la relación $T / T_{s}$ tiende a crecer sin límites a medida que $V \rightarrow 1 / \gamma(\gamma=1.4)$, y $r / r_{s} \rightarrow 0$. La liberación instantánea de la energía implica la existencia de una temperatura inicial cuyo valor no tiene límites. Si no se acepta el concepto de la temperatura inicial sin límites deberá, necesariamente, especificarse como es el proceso de liberación de la energía dentro de la burbuja. Lógicamente, el procedimiento de cálculo aquí expuesto ya no sería aplicable.

\section{CONCLUSIONES}

Se presenta un análisis basado en conceptos teóricos y aspectos numéricos conducente a la definición de burbujas explosivas creadas por instantáneas liberacion de energías. Dichas liberaciones energéticas son de tal magnitud que la expansión de la burbuja es muy rápida y las relaciones entre las variales físicas de ambos lados del choque que limita 
a la burbuja, convalidan las hipótesis que se hacen cuando las ondas de choque son fuertes. Esto posibilita a que las propiedades del gas contenido en el interior de la burbuja, puedan ser descritas utilizando metodologías que conducen a la obtención de soluciones similares. Aquí en particular, ha sido utilizada la formulada originalmente por Sedov y luego presentada según Hayes y Probstein para producir resultados en función del tiempo. Las variables y propiedades del flujo en el interior de la burbuja son necesarias para evaluar toda constante de proporcionalidad a utilizar cuando se pretende relacionar el radio asignado a la burbuja, con la energía liberada y con el tiempo que transcurre desde su generación hasta que alcance dicho radio. También lo serán cuando dados el radio de la burbuja y la velocidad de expansión, se quiere determinar la energía necesaria para su creación (Tabla 1).

El análisis efectuado sobre la distribución de la temperatura en el interior de la burbuja, confirma que la liberación instantánea de energía (es decir, sin especificar ningún proceso que depende del tiempo), exige una temperatura inicial sin límites.

\section{REFERENCES}

[1] L. Gutiérrez Marcantoni, S. Elaskar, J. Tamagno, and P. Bruel, "Simulación de ondas explosivas usando OpenFoam", Mecánica Computacional. vol 37, pp. 1075-1084

[2] L. Howarth, Modern Development in Fluid Dynamics, University Press, Oxford, 1956

[3] L. Sedov, Similarity and Dimensional Methods in Mechanics, CRC Press, 1993.

[4] W. Hayes, and R. Probstein, Hypersonic Flow Theory. Academic Press, 1959.

[5] J. Tamagno, W. Schulz, and S. Elaskar, Dinámica de los Gases. Flujo Unidimensional Estacionario. Topics in Applied and Computacional Mechanics, Asociación Argentina de Mecánica Computacional (AMCA). Ed. Universitas. Córdoba, 2008.

[6] J. Tamagno, G. Cid, S. Elaskar, and W. Schulz, Aplicaciones de la Dinámica de los Gases a Flujos Inestacionarios y Supersónicos. Ed. Universitas. Córdoba, 2013. 